\title{
Informations du secrétariat
}

Marianne Roth | Ursula Enggist | Claudia Menolfi

\section{EXCLUSIF POUR LES MEMBRES ASP}

\section{Procès-verbal de l'assemblée ordinaire} des membres du 24 mars 2018

Le procès-verbal en allemand, français et italien a été envoyé par mail à nos membres. Une version papier peut être commandée auprès du secrétariat et consultée sur le site Internet dans la rubrique des membres.

La prochaine assemblée des membres aura lieu le 23 mars 2019 à Zurich.

\section{Convention IV}

Les psychothérapies pour enfants et mineurs, les difficultés à la naissance et l'intégration professionnelle peuvent sous certaines conditions être couvertes par l'intermédiaire des Soins Intégrés (IV). Les membres de l'ASP reconnus au niveau fédéral disposant d'une autorisation d'exercer ont la possibilité sur demande d'adhérer à la convention entre l'Office fédéral des assurances sociales (OFAS) et l'ASP. Notre site Internet ou le bureau de l'ASP qui réceptionne aussi les demandes fournit des informations.

\section{Formulaires de décompte}

Pour la facturation de l'honoraire à leurs patientes ou patients, les membres peuvent commander auprès du secrétariat le modèle pratique et modifiable au format PDF ou le télécharger dans la rubrique protégée des membres sur le site Internet.

\section{Prestations et tarifs}

La notice contenant les directives de l'ASP concernant les prestations et tarifs est consultable dans la rubrique des membres de notre site Internet à la rubrique « Notices ».

\section{Liste des caisses maladie}

Savez-vous quelle caisse maladie rembourse quel montant pour la psychothérapie ? Demandez la liste des caisses maladie auprès du secrétariat ou informez-vous sur notre site Internet dans la rubrique « Téléchargements ».

\section{Les membres de l'ASP sont}

des messagers et messagères des professions dans le domaine de la psychothérapie

Profitez de l'occasion d'informer vos patientes et patients de votre reconnaissance fédérale et de la protection des titres.

Expliquez-leur leurs droits et obligations, le secret professionnel et la protection dont ils bénéficient en étant votre patiente ou patient.

\section{NOUVEAU : Adresse mail HIN cryptée}

Sécurisez votre adresse mail HIN personnelle cryptée à des conditions spéciales accordées aux membres de l'ASP. Vous garantissez ainsi un transfert protégé de vos données électroniques sensibles.

\section{Offres d'emploi et cabinets}

Consultez la rubrique « Actualités » sur notre site Internet. En plus d'informations actuelles et de messages des associations, vous trouverez les appels d'offres pour des postes vacants et des cabinets. Vous avez une offre ou souhaitez de plus amples renseignements ? Contactez le secrétariat pour obtenir de plus amples informations sur le placement sur notre site Internet.

\section{Inscription de nos membres dans PsyReg}

Tous nos membres ordinaires sont inscrits au PsyReg en tant que psychothérapeutes reconnus au niveau fédéral. Les nouveaux membres admis sont automatiquement communiqués à l'Office fédéral de la santé (OFS) pour inscription au registre. Vous devez envoyer vos souhaits de modification de votre inscription directement à l'OFS.

Les directions cantonales en charge de la santé sont compétentes pour l'inscription valable dans le PsyReg des autorisations cantonales d'exercer la profession.

\section{Devoir de formation continue des membres}

Les psychothérapeutes sont tenu(e)s légalement de participer constamment à des formations continues. Elles visent au perfectionnement scientifique, à létude de la théorie ainsi quà lélargissement et à l'approfondissement de compétences pratiques. Notre site Internet fournit de plus amples informations ainsi que notre programme complet de formation post-graduée à la rubrique « Formation post-graduée \& Formation continue».

\section{Publications et informations pertinentes en matière de psychothérapie}

Dans la rubrique des membres de notre site Internet, vous trouverez des publications qui sont contraignantes pour vous en tant que membre de l'ASP. En plus des règlements, les notices font référence à diverses questions sur des domaines spécifiques des professions relevant du domaine de la psychothérapie.

Utilisez notre secrétariat comme point d'informations et de renseignements.

\section{NOUVEAU : Archivage de votre documentation}

Envisagez-vous de vous retirer de la vie active et souhaitez-vous archiver vos dossiers en toute sécurité ? Voulez-vous être sûr(e) que votre documentation recueillie soit conservée en toute sécurité au sens du règlement de succession? L'ASP peut proposer en exclusivité à ses membres une solution abordable et sûre. Vous obtiendrez de plus amples renseignements auprès de notre secrétariat. 


\section{ASSURANCES}

\section{Pack Tout Compris}

Grâce au contrat collectif avec notre agence d'assurances, Swiss Quality Broker AG, nous sommes en mesure de proposer à nos membres un pack exclusif de prestations abordables en assurances, qui contient les offres suivantes :

- Caisse maladie

- Assurance responsabilité civile professionnelle

- Assurance protection juridique

- Assurances de choses

- Assurance d'indemnités journalières en cas de maladie ou d'accident

- Assurance pour perte de gain

Les membres de l'ASP peuvent également profiter auprès de ZURICH Versicherung de cotisations avantageuses pour leur assurance automobile, deux roues, assurance habitation, responsabilité individuelle et assurances des biens.

Renseignez-vous sur les détails sur notre site Internet dans la rubrique protégée des membres.

\section{Autre recommandation:}

- Caisse de retraite (deuxième pilier)

La Pro Medico Stiftung Zurich propose aux membres de l'ASP, en tant que caisse de prévoyance d'association du deuxième pilier, la possibilité d'une prévoyance professionnelle souple. Il s'agit d'offres financièrement attrayantes en matière de prévoyance retraite individuelle pour les travailleurs indépendants et leur personnel.

\section{INFORMATIONS GÉNÉRALES}

\section{www.psychotherapie.ch}

Notre site Internet contient des informations intéressantes pour nos membres dans une rubrique protégée par mot de passe. Les dates de la prochaine assemblée des membres, des colloques de la Conférence de la Charte, des solutions en matière d'assurances, etc. sont également disponibles dans cette rubrique. Nous recommandons de la consulter régulièrement. En plus d'informations sur notre organisation et sur la psychothérapie en général, le site Internet contient des informations pour le grand public sur les thèmes :

- Politique professionnelle \& développement professionnel

- Formation post-graduée \& formation continue

- Science \& recherche.

Des avis sur des manifestations en cours, des formations continues, des colloques, etc. sont disponibles sur notre site Internet à la rubrique « Agenda ».

\section{Actualités}

Informez-vous par exemple sur la version du modèle de la prescription médicale ainsi que sur d'autres thèmes brûlants sur notre site Internet à la rubrique " Actualités ». Avez-vous un souhait ou une suggestion? Pour ce faire, adressez-vous à notre bureau.

\section{PUBLICATIONS}

\section{Revue à jour ! - Développement professionnel en psychothérapie}

La revue à jour ! est l'organe bilingue (allemand et français) d'informations de l'ASP avec un contenu sur la politique professionnelle et la technique. Elle se veut être un lien entre nous en tant qu'association professionnelle et nos membres ainsi que toutes les lectrices et tous les lecteurs intéressés par la psychothérapie.

\section{Revue Psychotherapie-Wissenschaft}

La revue Psychotherapie-Wissenschaft est au service du développement de la psychothérapie. Des articles sur la pratique et la recherche favorisent léchange interdisciplinaire sur des questions fondamentales comme l'indication, la méthodologique et l'efficacité.

Deux numéros sont publiés deux fois par an. Par ailleurs, les articles des deux revues sont consultables sur Internet en version numérique à l'adresse : www.psychotherapie-wissenschaft.info

\section{Annonces}

Profitez de la possibilité de publier vos notifications ou annonces dans nos revues. Des psychothérapeutes praticien(ne)s ainsi que des professionnels issus de la science, la recherche et la pratique sont le public ciblé par les contributions publiées. Renseignez-vous auprès du bureau sur les possibilités de publication, les tarifs et les modes de parution.

\section{Newsletter}

Notre newsletter vous informe des activités en cours de l'association ainsi que des développements en matière de politique professionnelle. Elle est publiée quatre fois par an.

\section{ORGANISATIONNEL}

\section{Média}

Le bureau reçoit avec plaisir les demandes des journalistes. Notre association réunit des praticiennes et praticiens expérimentés sur différents thèmes et méthodes thérapeutiques, également actuels.

\section{Membre du conseil d'administration de l'ASP}

- Gabriela Rüttimann, Présidente

- Veronica Defièbre, vice-présidente, Direction Conférence de la Charte

- Nicola Gianinazzi, déléguée Suisse italienne

- Peter Schulthess, rédacteur en chef à jour ! et Psychotherapie-Wissenschaft 


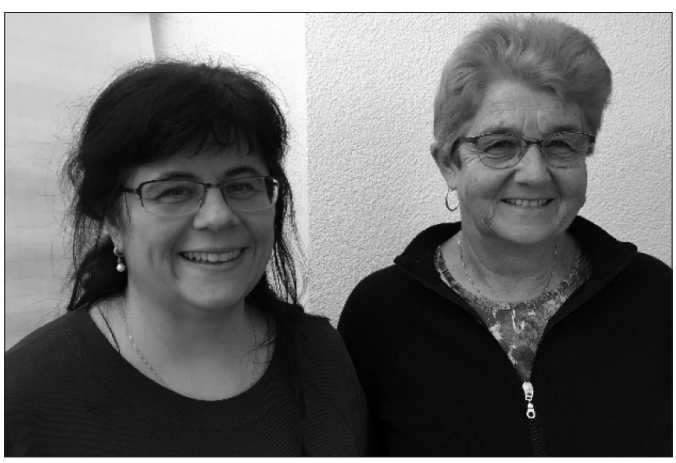

(de la gauche): Claudia Menolfi et Ursula Enggist

\section{Bureau}

- Marianne Roth, directrice générale

- Ursula Enggist, secrétariat

- Claudia Menolfi, secrétariat

Contact :

Téléphone : 0442689300

marianne.roth@psychotherapie.ch ursula.enggist@psychotherapie.ch claudia.menolfi@psychotherapie.ch

\section{Attualità dalla Svizzera italiana Psicologia, Biomedicina e Supercalcolo neuroscientifico in avvicinamento Nicola Gianinazzi}

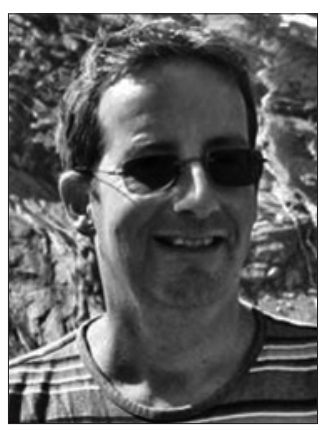

In accordo con il Comitato il mio ruolo si rafforza sempre più quale antenna di comunicazione tra la Svizzera italiana e le altre parti elvetiche.

Inoltre come delegato collaboro intensamente con l'IRG nel preparare e accompagnare le fasi conclusive dell'accreditamento definitivo: in particolare si è trattati di accompagnare anche la visita all'IRG degli esperti designati dall'AAQ.

Da quest'anno inoltre - entrando a far parte della redazione di Psychotherapie-Wissenschaft - la Svizzera italiana e il panorama italiano saranno più presenti e visibili nella nostra rivista tematica. Si è infine ulteriormente approfondito il lavoro di contatto con docenti universitari di varie università e facoltà ticinesi. Questa interessante attività di public relations è utile e necessaria per arricchire sia le proposte formative, sia i contributi delle nostre riviste associative, sia le prospettive di sviluppo della didattica e della ricerca. $\hat{E}$ in questo ambito che si approfondisce pure la collaborazione redazionale con la rivista Psicoterapia e scienze umane (www.psicoterapiaescien zeumane.it), di cui è co-direttore Paolo Migone.

\section{Panorama ticinese}

A partire dall'autunno 2020 all'USI inizieranno i corsi della Facoltà di Scienze biomediche, che nasce a Lugano in collaborazione con le Università di Basilea e Zurigo, nonché con il Politecnico di Zurigo (ETH). Essa lavorerà in modo sinergico con altre Facoltà dell'USI in campi quali la comunicazione sanitaria, la gestione e l'economia della sanità, l'applicazione medica della scienza computazionale e dovrebbe pure includere la psichiatria.

Particolarmente affini al nostro ambito psicoterapeutico saranno materie - magari potenziali corsi di aggiornamento per noi psicoterapeuti - inerenti alle seguenti tematiche nel campo della relazione e della comunicazione in ambito sanitario (secondo quello che personalmente definirei il «Nuovo modello modulare di Scienze Psicoterapeutiche»):

Retorica, argomentazione e persuasione/La comunicazione del rischio/La promozione della salute e le campagne mediatiche/Processi e tecnologie della comunicazione/Management della comunicazione sanitaria.

Non bisogna infine dimenticare gli interessantissimi corsi su filosofia della mente ed epistemologia tenuti nel nuovo Master of Arts di Filosofia dell'USI, all'Istituto di Studi Filosofici (ISFI) o direttamente alla Facoltà di Teologia di Lugano (FTL).

Inoltre proprio a Lugano si trova il Centro Svizzero di Calcolo Scientifico (CSCS) del Politecnico di Zurigo (ETH). 